\title{
Covariation of Activity in Habenula and Dorsal Raphé Nuclei Following Tryptophan Depletion
}

\author{
J . S. Morris,* K. A. Smith, † P. J . Cowen,† K. J . Friston,* and R. J . Dolan*,, 1 \\ *Well come Department of Cognitive Neurology, 12 Queen Square, London, WC1N 3BG, United Kingdom; †U niversity Department of \\ Psychiatry, Warneford Hospital, Oxford, OX3 7J X, United Kingdom; and ¥Royal Freeand University College Hospitals School of M edicine, \\ Rowland Hill Street, London NW3 2DF, United Kingdom
}

Received December 9, 1998

\begin{abstract}
Abnormal serotonergic function is implicated in the pathogenesis of affective disorders. We induced transient depressive relapses in volunteer patients by rapidly depleting plasma tryptophan, the precursor of serotonin (5-HT), and measured neural activity during different cognitive tasks using positron emission tomography (PET). Neural activity in several 5-HT-related brain areas, e.g., dorsal raphé, habenula, septal region, amygdala, and orbitofrontal cortex, covaried significantly with plasma levels of tryptophan and ratings of depressed mood. Task-specific responses in left amygdala and left anterior cingulate were attenuated by tryptophan depletion. We used these PET data to test the hypothesis that projections from the habenula modulate dorsal raphé activity and that this modulation is enhanced in patients experiencing a profound mood change following serotonergic challenge. A strong linear correlation $\left(r^{2}>0.5\right)$ between habenula and raphé activity was observed in subjects with postdepletion ratings $\geq 10$ on a modified Hamilton depression scale, whereas subjects experiencing mi Ider changes in mood had weaker habenula-raphé coupling $\left(r^{2}<0.5\right)$. These data support a model of the serotonergic system in which the habenula projection to the raphé represents a convergent feedback pathway that controls the release of 5-HT throughout the brain. In our experiment we were able to engage this system in patients who were sensitivetotryptophan depletion. $\odot 1999$ Academic Press

Key Words: habenula; dorsal raphé; tryptophan; serotonin; depression.
\end{abstract}

\section{INTRODUCTION}

The midbrain raphé nuclei are the principal source of cerebral serotonin (Tork and Hornung, 1990). However, despite strong evidence that a disturbance of serotonergic function underlies human mood disorders, the

${ }^{1}$ To whom correspondence should be addressed. Fax: $+44(0) 171$ 813 1420. E-mail: r.dolan@fil.ion.ucl.ac.uk. involvement of the raphé nuclei in the pathophysiology of depression remains unclear (Coppen, 1967; Stanley and Mann, 1983; Heninger et al., 1984; Cowen and Charig, 1987; Meltzer, 1990). One of the few forebrain structures projecting to the dorsal raphé is the habenula nucleus in the epithalamus (Aghajanian and Wang, 1977; Sakai et al., 1977). Animal experiments have shown that the habenula is able to influence the activity of serotonergic neurons in the raphé (Wang and Aghajanian, 1977) and modulate release of cerebral serotonin (Soubrie et al., 1977; Reisne et al., 1982). Animal data have also implicated the habenula in the expression of depressed behavior and the action of antidepressant drugs (Thornton et al ., 1985; Cal decottHazard et al., 1988). Despite this evidence that habenular-raphé interactions have an important role in both mood regulation and the etiology of depression, there have been no previous reports of abnormal neural activity in either the habenula or the raphé nuclei in the context of human affective disorder.

The serotonergic hypothesis of mood disorder has received strong empirical support from experiments in which transient depressive relapses are induced by a reduction in the level of plasma tryptophan, the amino acid precursor of serotonin (5-HT) (Del gado et al., 1990; Smith et al., 1997). In the present study, we combined the technique of tryptophan depletion with positron emission tomography (PET) to measure neural activity associated with alterations of mood in recovered depressed patients during the performance of specific psychological tasks (verbal fluency and word repetition). We report here the results of analyses that identify neural responses directly related to changes in tryptophan level, mood, and cognitive task. Mood and task-related activity occurring independently of changes in tryptophan level have been reported separately (Smith et al., 1998).

\section{METHODS}

Subjects. Eight male subjects (mean age 39.1 years, range 27-64 years) were recruited from psychiatric 
outpatients clinics. All subjects had suffered at least two episodes of major depression (DSM-III-R criteria), but were in full remission at thetime of thestudy. Six of the subjects were on long-term (>6 months) antidepressant treatment. The drugs taken by the subjects (in various individual combinations) included sertraline, paroxetine, and fluoxetine (selective serotonin reuptake inhibitors or SSRIs); trazodone (a nonselective serotonin reuptake inhibitor); phenelzine and tranylcypromine (monoamine oxidase inhibitors or MAOIs); amitriptyline (a tricyclic); and lithium. The two medication-free subjects (subjects 1 and 2 in Table 1) had both responded to fluoxetine (an SSRI) during previous depressive episodes. Drug regimes did not change during the study. All subjects gave informed consent and were told explicitly that the procedure may result in the return of some, or all, of their depressive symptoms. The study was approved by the research ethics committee at the National Hospital and by the UK Administration of Radioactive Substances Advisory Committee.

I mageacquisition. Scans of the distribution of $\mathrm{H}_{2}{ }^{15} \mathrm{O}$ were obtained using a Siemens/CPS ECAT EXACT $\mathrm{HR}+\mathrm{PET}$ scanner operated in high-sensitivity 3-D mode. Subjects received a total of $350 \mathrm{MBq}$ of $\left[{ }^{15} \mathrm{O}\right] \mathrm{H}_{2} \mathrm{O}$ over 20 s through a forearm cannula for each scan, and activity was measured during a 90-s time window. The start of scanning window was triggered automatically when a preset threshold was reached ( $3 \times 10^{6}$ counts). Each subject had two sessions of 6 scans (i.e., a total of 12 scans). The PET images comprised $2 \times 2 \times 3-\mathrm{mm}$ voxels with a $6.4-\mathrm{mm}$ transaxial and $5.7-\mathrm{mm}$ axial resolution (full width at half-maximum). The data were analyzed with statistical parametric mapping (SPM 96, Well come Department of Cognitive Neurology, London, UK; http://www.fil.ion.ucl.ac.uk/spm) implemented in Matlab (Mathworks, Sherborn, MA). Structural MRIs from each subject were coregistered to the PET data following realignment of the PET time series. All the scans were then transformed into a standard stereotaxic space (Talairach and Tournoux, 1988; Friston et al., 1995). Following this normalization, the scans were smoothed using a Gaussian filter set at $12-\mathrm{mm}$ full width at half-maximum. TherCBF measurements were adjusted to a gl obal mean of $50 \mathrm{ml} / \mathrm{dl} / \mathrm{min}$.

Tryptophan depletion protocol. Subjects were scanned on 2 days separated by at least 1 week. At 0900 on one of the scanning days (balanced day), subjects received a nutritionally balanced mixture of 16 amino acids (L-alanine, L-arginine, L-cysteine, glycine, Lhistidine, L-isoleucine, L-leucine, L-lysine monohydrochloride, L-methionine, L-phenylalanine, L-proline, Lserine, L-threonine, L-tryptophan, L-tyrosine, and L-valine) made up in $200 \mathrm{ml}$ of water flavored with black currant; at 0900 on the other scanning day (depleted day) subjects were given an identical mixture except that the tryptophan was omitted. The tryptophan-depleted and balanced drinks were given according to a double-blind placebo-controlled protocol. The order of administration was balanced and randomly allocated across the subjects. Prior to both scanning days, subjects had a $24-\mathrm{h}$ low protein diet and ate a light lunch of low tryptophan foods at 1300. After scanning, which took place between 1400 and 1500, they resumed a normal diet. Blood samples were taken for measurement of total and free tryptophan immediately before ingestion of the mixture (0900) and immediately after scanning (1500).

Plasma total and free tryptophan levels were measured by high-performance liquid chromatography (HPLC) with amperometric end-point detection. The tryptophan was separated from plasma by deproteinization with $10 \%$ trichloroacetic acid followed by centrifugation at high speed. The residual clear supernatant was diluted with mobile phase and injected into the HPLC system via an automatic injector. Tryptophan was separated from plasma proteins by forcing the plasma through a filter with a molecular weight cutoff of 10,000 Da, using centrifugation. The residual clear aqueous liquid was injected directly into the HPLC system. Stock standards and quality control samples were prepared. Quality control samples were processed in the same way as the samples. Standards were prepared for each assay from stock and injected directly into the HPLC system. The intra- and interassay coefficients of variation were 8.7 and $9.9 \%$ for the total tryptophan assay and 10.6 and $11.4 \%$ for the free tryptophan assay, respectively. The assay sensitivity was $0.05 \mu \mathrm{g} / \mathrm{ml}$, with a signal to noise ratio of 3:1.

Clinical rating. Following the administration of the amino acid mixtures, subjects remained in a quiet environment, being allowed to read only neutral material. Ratings of depressive symptoms were made at 0845, 1330, and 1530 by an observer blind to the mixture allocation. A modified version of the Hamilton rating scale for depression (HAM-D) was used which excluded five items that could not change during the course of the experiment (insomnia-initial, middle, or delayed; genital symptoms; and weight loss). In addition, subjects rated their own mood state at 1330 using the Beck Depression Inventory, and during scanning they rated their levels of happiness, sadness, and anxiety according to a $100-\mathrm{mm}$ visual analogue scale. Each of the subjects was clinically reassessed later on the same evening and al so the following morning.

Psychological tasks. During scanning, subjects performed either (a) a paced word repetition task, in which words were spoken by the experimenter every $5 \mathrm{~s}$ and immediately repeated by the subject, or (b) a paced orthographic verbal fluency task, in which a letter was repeated every $5 \mathrm{~s}$ by the experimenter and subjects generated a word beginning with that letter for each 
repetition. If the subject could not generate a word, he said "pass" and was given a new letter that was repeated in the same way. A new letter was automatically introduced after 10 repeats of a single letter. The tasks were alternated across the six scans in each session, with half the subjects having theorder $A B A B A B$ and half BABABA.

Statistical analysis. A blocked (by subject) ANCOVA model was fitted to the data at each voxel, with a condition effect for each psychological task in each of the depleted or balanced sessions and global CBF as a confounding covariate. Predetermined contrasts of the condition effects at each voxel were assessed for individual subjects using the usual t statistic, and conjunction analyses were performed across the eight subjects to give a final statistical parametric map (SPM) for the contrast. This procedure was undertaken to overcome the limited generalizability of the fixed effects model employed. Measures of plasma tryptophan and Hamilton depression ratings obtained pre- and postscanning were used to generate covariates of interest for separateanalyses. Individual values for each of the 12 scans were produced by linear interpolation of both variables across the scanning session.

Following these provisional analyses, the adjusted rCBF values for the maximal focus of activation in the habenula $(x=4, y=-24, z=6$ ) were used as a covariate of interest to identify psychophysiological interactions, i.e., context-specific changes in the contribution of one brain region to explaining the activity in another (Friston et al., 1997). The habenula was chosen as the maximally activated region in the Hamilton covariate analysis (Table 2a). Each subject was assigned a separate value representing his Hamilton score on the depletion day, and a mean-corrected, modified Hamilton score-weighted, subject-specific regression analysis was performed using habenula rCBF as a covariate of interest and subject blocks as a confound. By using the appropriate contrast to test for differences in the regression of regional activity on habenula activity, the resulting SPM(t) identified those voxels, across the entire brain, in which the contribution of habenula activity varied as a function of posttryptophan depletion Hamilton score. The general methods employed by SPM are described in detail by Friston et al. (1995).

\section{RESULTS}

\section{Pharmacological}

There were large decreases in total plasma tryptophan level for all subjects on the depletion day (mean change over $6 \mathrm{~h},-70 \%)$, compared to a small mean increase $(+2 \%)$ on the balanced day. The difference in total tryptophan levels on the 2 days was significant on a paired t test $(P<0.001)$. Free tryptophan levels also showed decreases on the depletion day (as much as 90\% in some subjects) but unfortunately, measurements in one subject were affected by inadequte separation of interference peaks, making the values unreliable (Table 1). Consequently, total tryptophan levels, constituting a complete and reliable set of biochemical data, were used as covariates of interest in the analysis of rCBF data.

Brain areas in which activity positively covaried with total tryptophan level included right middle temporal gyrus, left inferior parietal cortex, right superior frontal gyrus, left orbitofrontal cortex, left amygdala, the septal region, and the dorsal pons (Table 2a). Right orbitofrontal cortex, right amygdala, dorsal raphé, right cerebellum, and left inferior parietal cortex negatively covaried with total tryptophan level (Table 2b). The most significant negative covariation with tryptophan level was recorded in a region immediately superior and lateral to the posterior commissure $(x=4, y=-28$, $z=6$ ). The anatomical location of this area corresponds precisely to that of the habenula nucleus in the epithalamus (Fig. 1) (Duvernoy, 1991; Mai et al., 1997).

Despite the small size of the habenula, and the spatial normalization and smoothing applied to the rCBF data, the identification of the habenula as a focus of neural activity in this experiment can be justified on

\section{TABLE 1}

Values of Free and Total Plasma Tryptophan (in $\mu \mathrm{g} / \mathrm{ml}$ ) for Each Subject

\begin{tabular}{|c|c|c|c|c|c|}
\hline \multirow[b]{3}{*}{ Subject } & \multirow[b]{3}{*}{$\begin{array}{l}\text { L-TP } \\
\text { assay }\end{array}$} & \multicolumn{2}{|c|}{ Balanced day } & \multicolumn{2}{|c|}{ Depleted day } \\
\hline & & $\begin{array}{l}\text { Predrink } \\
\text { L-TP }\end{array}$ & $\begin{array}{c}\text { Postscan } \\
\text { L-TP }\end{array}$ & $\begin{array}{c}\text { Predrink } \\
\text { L-TP }\end{array}$ & $\begin{array}{c}\text { Postscan } \\
\text { L-TP }\end{array}$ \\
\hline & & $\begin{array}{l}\text { concn } \\
(\mu \mathrm{g} / \mathrm{ml})\end{array}$ & $\begin{array}{l}\text { concn } \\
(\mu \mathrm{g} / \mathrm{ml})\end{array}$ & $\begin{array}{l}\text { concn } \\
(\mu \mathrm{g} / \mathrm{ml})\end{array}$ & $\begin{array}{l}\text { concn } \\
(\mu \mathrm{g} / \mathrm{ml})\end{array}$ \\
\hline \multirow[t]{2}{*}{1} & Total & 15.5 & 13.9 & 15.3 & 2.3 \\
\hline & Free & 0.46 & 0.68 & 0.53 & 0.04 \\
\hline \multirow[t]{2}{*}{2} & Total & 8.0 & 16.3 & 11.1 & 2.09 \\
\hline & Free & a & a & a & a \\
\hline \multirow[t]{2}{*}{3} & Total & 15.9 & 13.5 & 15.6 & 2.3 \\
\hline & Free & 0.55 & 0.97 & 0.64 & 0.13 \\
\hline \multirow[t]{2}{*}{4} & Total & 11.6 & 13.7 & 11.5 & 1.1 \\
\hline & Free & 0.6 & 1.09 & 0.53 & 0.03 \\
\hline \multirow[t]{2}{*}{5} & Total & 13.2 & 15.5 & 14.4 & 2.8 \\
\hline & Free & 0.86 & 1.35 & 0.49 & 0.06 \\
\hline \multirow[t]{2}{*}{6} & Total & 15.5 & 23.2 & 13.3 & 3.8 \\
\hline & Free & 0.73 & 2.2 & 0.39 & 0.04 \\
\hline \multirow[t]{2}{*}{7} & Total & 15.8 & 13.5 & 13.2 & 2.2 \\
\hline & Free & 0.46 & 1.09 & 0.53 & 0.15 \\
\hline \multirow[t]{2}{*}{8} & Total & 17.9 & 12.9 & 14.9 & 1.6 \\
\hline & Free & 2.6 & 0.62 & 3.6 & 2.9 \\
\hline
\end{tabular}

Note. Predrink measurements were made at 0900, immediately before ingestion of the balanced or depleted amino acid mixtures. Postscan measurements were made at 1500. The balanced and depleted sessions were separated by at least 1 week, with doubleblind counterbalancing of the order across subjects.

a In this subject the free tryptophan levels were unreliable due to a failure of separation of interference peaks. 
a number of grounds. First, the habenula is known to be a crucial serotonergic structure (Aghajanian and Wang, 1977; Sakai et al., 1977; Wang and Aghajanian, 1977; Soubrie et al., 1977; Reisne et al., 1982), making it biologically plausible that activity in this nucleus, rather than in neighboring midline regions, is modulated by plasma tryptophan depletion. Second, although spatial normalization may slightly distort the absolute dimensions of brain scans of individual subjects, it will not alter the consistent and closetopographical relationship of the habenula to the fixed anatomical landmark of the posterior commissure. Finally, although the rCBF data are spatially smoothed with a 12-mm Gaussian filter, the resolution of the maxima (i.e., the reported peaks in Tables 2-4) is considerably more precise, in the range of 1-2 mm. Smoothed PET data have been used successfully, for example, in producing retinotopic maps of human visual cortex (Fox et al., 1987).

\section{Psychological}

Increases in Hamilton depression ratings occurred in seven of the eight subjects after the tryptophandepleted drink: four subjects had ratings of $\geq 10$ points on the modified scale, while three rated between 5 and 9 points (Fig. 3). Following the balanced drink, no subjects had increases in Hamilton score of $>5$ points

TABLE 2

Covariation of rCBF and Plasma Tryptophan Level

\begin{tabular}{ccc}
\hline & & $Z$ \\
Area & Coordinates & scores \\
\hline
\end{tabular}

(a) Brain regions exhibiting a significant positive covariation with plasma tryptophan level ( $P<0.001$, uncorrected)

Right middle temporal gyrus Left inferior parietal cortex

Right superior frontal gyrus

Left orbitofrontal gyrus

Left amygdala

Dorsal pons

Septal region

$\begin{array}{cl}60,-8,-22 & 6.02^{*} \\ -44,-50,44 & 5.67^{*} \\ 16,48,44 & 5.44 \\ 0,28,-18 & 5.10 \\ -26,22,-16 & 4.94 \\ -28,-4,-18 & 4.63 \\ -2,-40,-24 & 3.79 \\ -4,18,2 & 3.15\end{array}$

(b) Brain regions exhibiting a significant negative covariation with plasma tryptophan level ( $\mathrm{P}<0.001$, uncorrected)

\begin{tabular}{lcl} 
Habenula & $4,-24,6$ & $5.69 *$ \\
Right cerebellum & $20,-58,-36$ & 5.22 \\
Dorsal raphé nucleus & $-10,-22,-16$ & 4.89 \\
Left inferior parietal cortex & $-32,-24,34$ & 4.85 \\
Right orbitofrontal gyrus & $24,28,-26$ & 4.32 \\
\hline
\end{tabular}

Note Each scan was assigned an individual value by linear interpolation between the plasma tryptophan measurements obtained before and after the scanning sessions. Coordinates of the maximally activated voxel and $Z$ scores are shown for each region. Maxima that survive correction for multiple comparisons $(P<0.001)$ are denoted by an asterisk.

\section{TABLE 3}

Covariation of rCBF and Hamilton Depression Rating

Area

Coordinates

Z

score

(a) Brain regions exhibiting a significant positive covariation with modified Hamilton depression rating ( $P<0.001$, uncorrected)
Habenula
Left parahippocampal gyrus
Right fusiform gyrus
Hypothalamus
Right putamen
Right parahippocampal gyrus
Dorsal midbrain
Right amygdala

$\begin{array}{cc}6,-24,4 & 5.79 \\ -4,-2,-28 & 5.08 \\ 18,-60,-14 & 4.96 \\ -4,0,-10 & 4.89 \\ 26,-16,8 & 4.84 \\ & \\ 28,-14,-26 & 4.65 \\ -12,-32,-12 & 3.25 \\ 26,-10,-26 & 3.09\end{array}$

(b) Brain regions exhibiting a significant negative covariation with modified Hamilton depression rating $(P<0.001$, uncorrected)

Left middle temporal gyrus

Right temporal pole

Dorsal pons

Left inferior parietal cortex

Right superior frontal gyrus

Septal region

$\begin{array}{cl}-44,-48,24 & 5.58^{*} \\ 36,20,-40 & 5.46 \\ -4,-38,-30 & 5.00 \\ -30,-64,42 & 4.38 \\ 18,44,38 & 4.35 \\ 4,20,0 & 3.78\end{array}$

Note. Each scan was assigned an individual value by linear interpolation between the ratings obtained before and after the scanning sessions. Coordinates of the maximally activated voxel and $Z$ scores are shown for each region. Maxima that survive correction for multiple comparisons ( $<0.001$ ) are denoted by an asterisk.

(mean change $=+0.75$ points). Brain regions in which activity positively covaried with Hamilton depression rating included habenula, right amygdala, bilateral parahippocampal gyri, and hypothalamus (Table 3a). The septal region was among several brain areas in which activity covaried negatively with Hamilton ratings (Table 3b).

\section{Psychophysiological}

In this regression analysis, we identified brain areas in which activity covaried with habenula responses as a function of subjects' postdepletion depression rating. Critically, activity in a region of the midbrain, comprising the dorsal raphé nucleus, exhibited an increasingly positive covariation with habenula responses as a function of postdepletion depression score (Fig. 2). Bivariate regression plots of habenula and raphé responses for each subject are shown in Fig. 3. Highly correlated responses $\left(r^{2}>0.5\right)$ are evident for subjects with a Hamilton score $\geq 10$, but less correlated activity $\left(r^{2}<0.5\right)$ for subjects with lower depression ratings. Other (unpredicted) brain areas with a similar covariation are shown in Table 4.

\section{Psychopharmacol ogical}

Subjects' performance on the paced verbal fluency and word repetition tasks did not differ significantly 
between the depleted and balanced days. Brain regions with increased activity on both days in the verbal fluency task relative to word repetition included left orbitofrontal cortex and left anterior cingulate gyrus. Areas with decreased activity in verbal fluency relative to word repetition included bilateral superior temporal gyri and bilateral amygdalae. These task-related changes accord with previous functional imaging studies in normal subjects and depressed patients (Baker et al., 1997; Drevets et al., 1995). Examining the interaction between serotonergic manipulation and cognitive task revealed that differential activity during the verbal fluency task was significantly reduced ( $P<0.01$, uncorrected) in both the left anterior cingulate $(x=-2$, $y=22, z=32)$ and the left amygdala $(x=-28, y=-6$, $z=-26)$ following tryptophan depletion.

\section{DISCUSSION}

The number of different brain regions modulated by plasma tryptophan depletion demonstrates the widespread influence of 5-HT on cerebral function (Table 2). Many of the activations, e.g., in the temporal and parietal lobes, were unexpected and may reflect how neural responses related to the general cognitive and attentional demands of the ongoing explicit tasks are affected by plasma tryptophan level. This discussion, however, focusses primarily on the neural responses of serotonergic structures, in particular, the habenula and dorsal raphé nuclei, since interpretation of the imaging data can be guided by models derived from animal experiments.

Activity in regions receiving strong serotonergic projections, e.g., left amygdala, septum, and left orbitofrontal cortex (Table 1a), covaried positively with levels of total plasma tryptophan, indicating decreased synaptic activity in these areas following tryptophan depletion. These data accord with a fluorodeoxyglucose (18F) PET study of tryptophan depletion which measured metabolic activity in predetermined regions of interest (Bremner et al., 1997). By contrast, responses in the dorsal raphé and habenula nuclei showed a negative covariation, i.e., an increased drive to these areas with decreased total tryptophan level (Table $2 b$ ). The main inputs to the habenula are fibers returning from serotonergic target regions (Marburg, 1944; Sutherland, 1982), while the habenula itself supplies the main
TABLE 4

Hamilton Score-Weighted Regression of Habenula Activity

\begin{tabular}{lcc}
\hline \multicolumn{1}{c}{ Area } & Coordinates & Z \\
\hline Right insula & $32,-12,0$ & $6.73^{*}$ \\
Left superior occipital gyrus & $-24,-78,34$ & $5.63^{*}$ \\
Dorsal raphé nuclei & $0,-28,-16$ & 5.03 \\
\hline
\end{tabular}

Note. Brain regions exhibiting a covariation with habenula activity (maximal voxel $x=4, y=-24, z=6$ ) that varied significantly as a function of subject's depression rating foll owing tryptophan depletion ( $P<0.001$, uncorrected). Habenula rCBF was used as a covariate of interest in a subject-specific regression analysis that was weighted by the modified Hamilton rating at the end of the tryptophan depletion scanning session. Coordinates of the maximally activated voxel and $Z$ scores are shown for each region. Maxima that survive correction for multiple comparisons ( $\mathrm{P}<0.001$ ) are denoted by an asterisk.

forebrain projection to the raphé (Aghajanian and Wang, 1977; Sakai et al., 1977). On the basis of this anatomical connectivity and other functional animal data (Wang and Aghajanian 1977; Soubrie et al., 1977; Reisne et al., 1982), it has been proposed that the habenula represents a crucial point of convergence in a feedback loop that controls raphéactivity (Nauta, 1958; Wang and Aghajanian, 1977; Stern et al., 1981). Our data, showing increased drive to the habenula and raphéin the presence of decreased activity in serotonergic projection fields, are consistent with this feedback hypothesis.

In vivo microdialysis data in animals provide evidence for both excitatory and inhibitory (GABAergic) projections from habenula to dorsal raphé. Activation of presynaptic GABA receptors in the dorsal raphé is thought to reduce responses to excitatory amino acids (Nishikawa and Scatton, 1985; Kalen et al., 1989). Alterations in the balance of these excitatory and inhibitory influences may account for the mooddependent changes in covariance between habenula and raphé identified in the present study (Table 4; Fig. 3). Subjects whose modified Hamilton depression rating was $\geq 10$ following tryptophan depletion showed a strong linear correlation $\left(r^{2}>0.5\right)$ between habenula and raphé activity, suggesting that excitation dominates over inhibition in the habenula-raphé pathway during depressed mood (Figs. 3a-3d). In subjects with lower $(<10)$ ratings, a weaker correlation was ob-

FIG. 1. A statistical parametric map (SPM) showing an epithalamic activation that encompasses the habenula nucleus (maximal voxel $x=4, y=-24, z=6$ ). The SPM is the result of an analysis identifying brain regions that covary negatively with plasma tryptophan level (i.e., show enhanced responses to increasing depletion). The activation is displayed on orthogonal sections of a canonical MRI, centered on the maximal habenula voxel at a threshold of $P<0.001$, uncorrected.

FIG. 2. A statistical parametric map (SPM) showing a dorsal midbrain activation that encompasses the dorsal raphé nucleus (maximal voxel $x=0, y=-28, z=-16$ ). The SPM is the result of a regression analysis that identifies brain regions in which the contribution of habenula activity increases as a function of subject's depressed mood following tryptophan depletion. Theactivation is displayed on orthogonal sections of a canonical MRI, centered on the maximal dorsal raphé voxel, and at a threshold of $\mathrm{P}<0.001$, uncorrected. 
sagittal

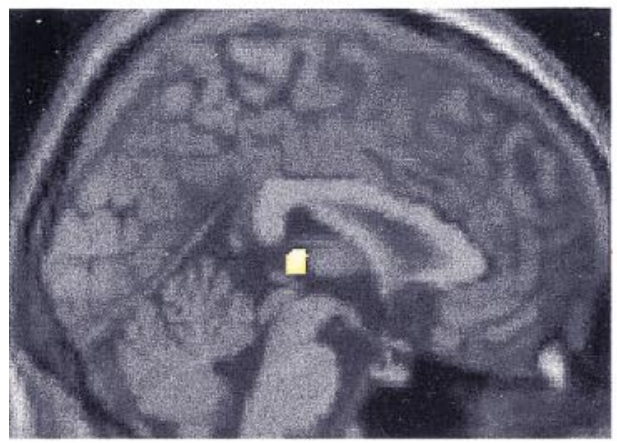

transverse

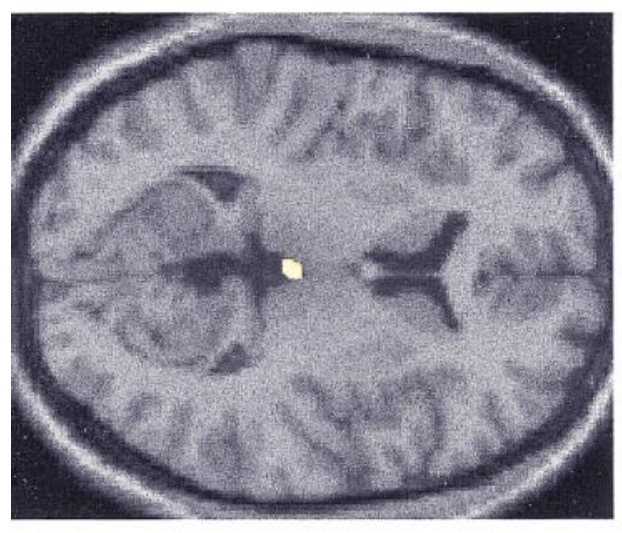

sagittal

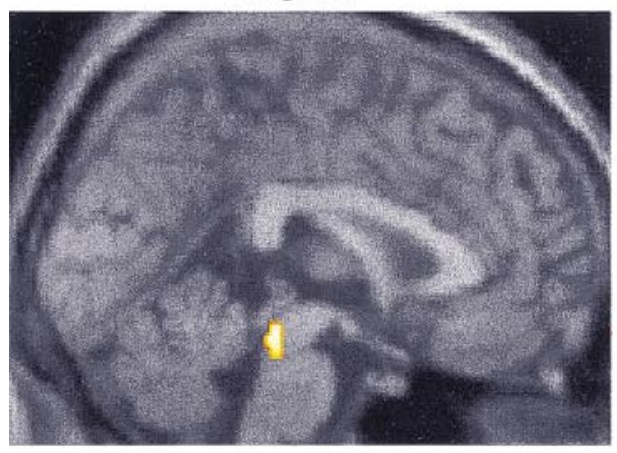

transverse

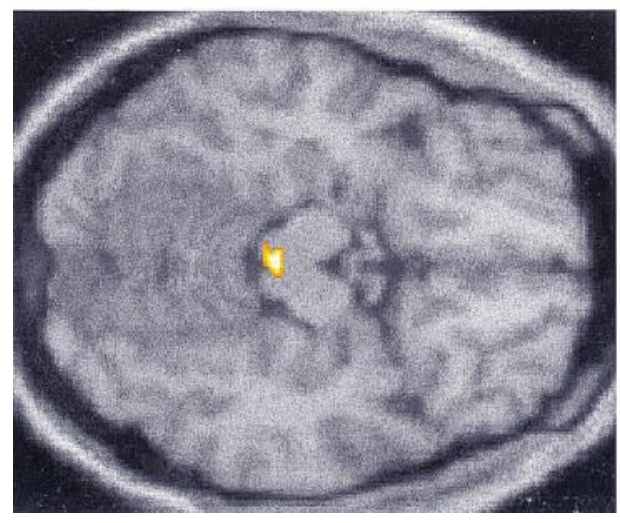

coronal
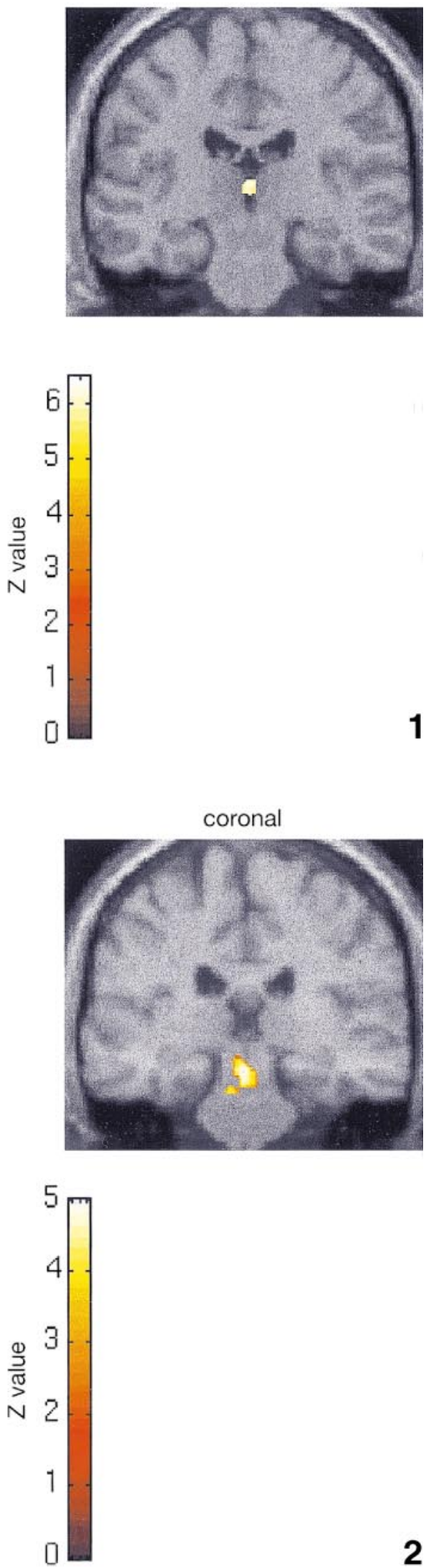

2 


\section{coronal}

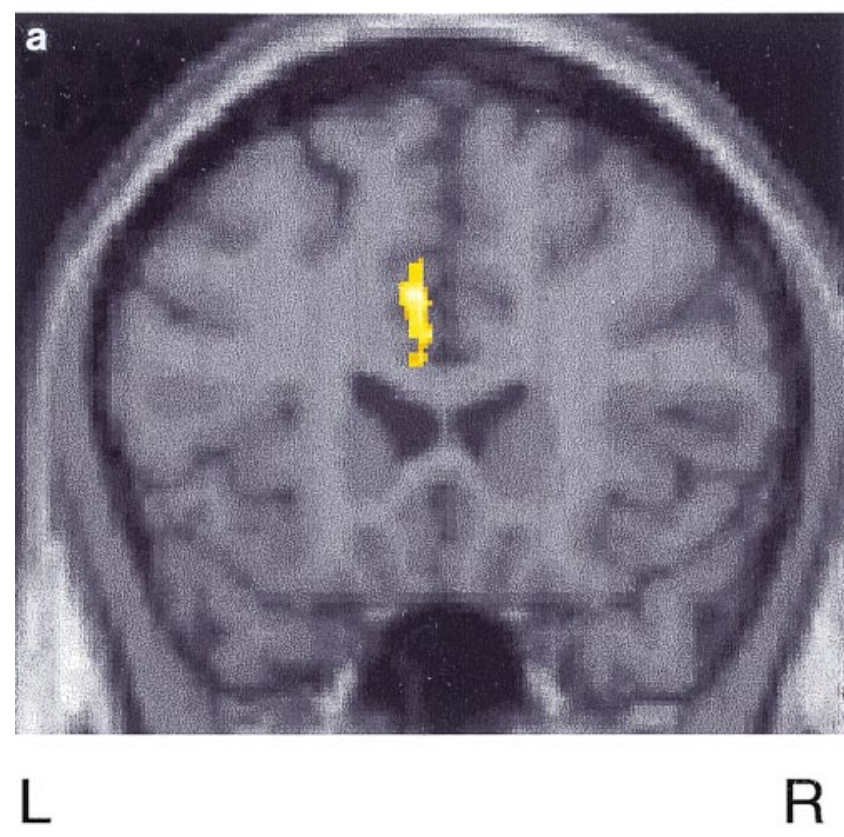

\section{coronal}

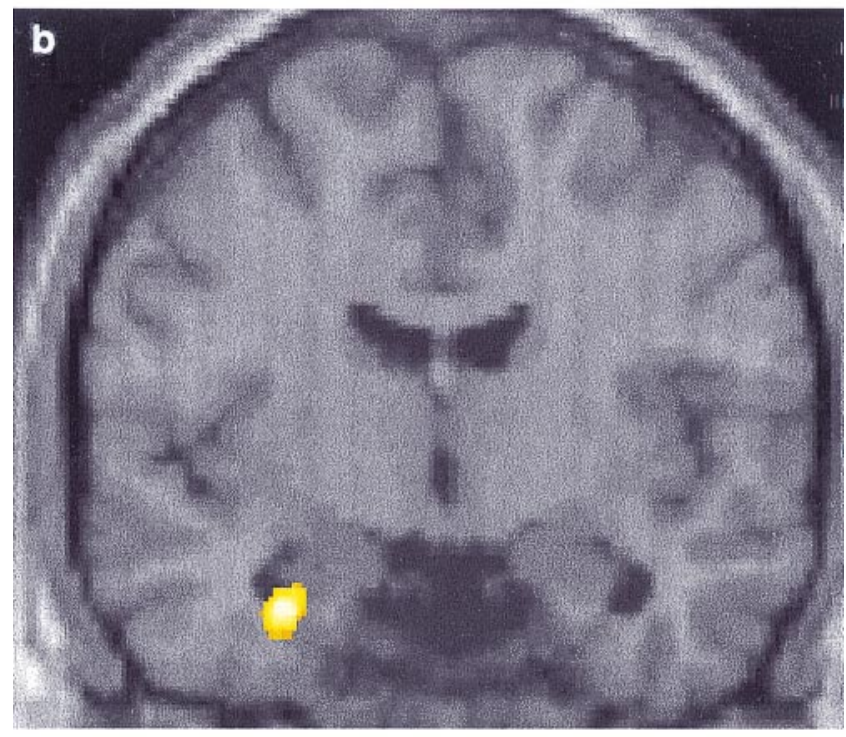

$\mathrm{L}$

$\mathrm{R}$

FIG. 4. (a) A statistical parametric map (SPM) showing activation of the left anterior cingulate gyrus (maximal voxel $x=-2$, $y=22$, $z=32$ ). The SPM is the result of a contrast identifying brain regions in which an enhanced response in the verbal fluency task is significantly attenuated by tryptophan depletion. The activation is displayed on a coronal section $(y=22)$ of a canonical $\mathrm{MRI}$, at a threshold of $\mathrm{P}<0.05$. (b) A statistical parametric map (SPM) showing activation of the left amygdala (maximal voxel $x=-28, y=-6, z=-26$ ). The SPM is the result of a contrast identifying brain regions in which a decreased response in the verbal fluency task is significantly attenuated by tryptophan depletion. The activation is displayed on a coronal section $(y=-6)$ of a canonical $M R I$, at a threshold of $P<0.01$. 

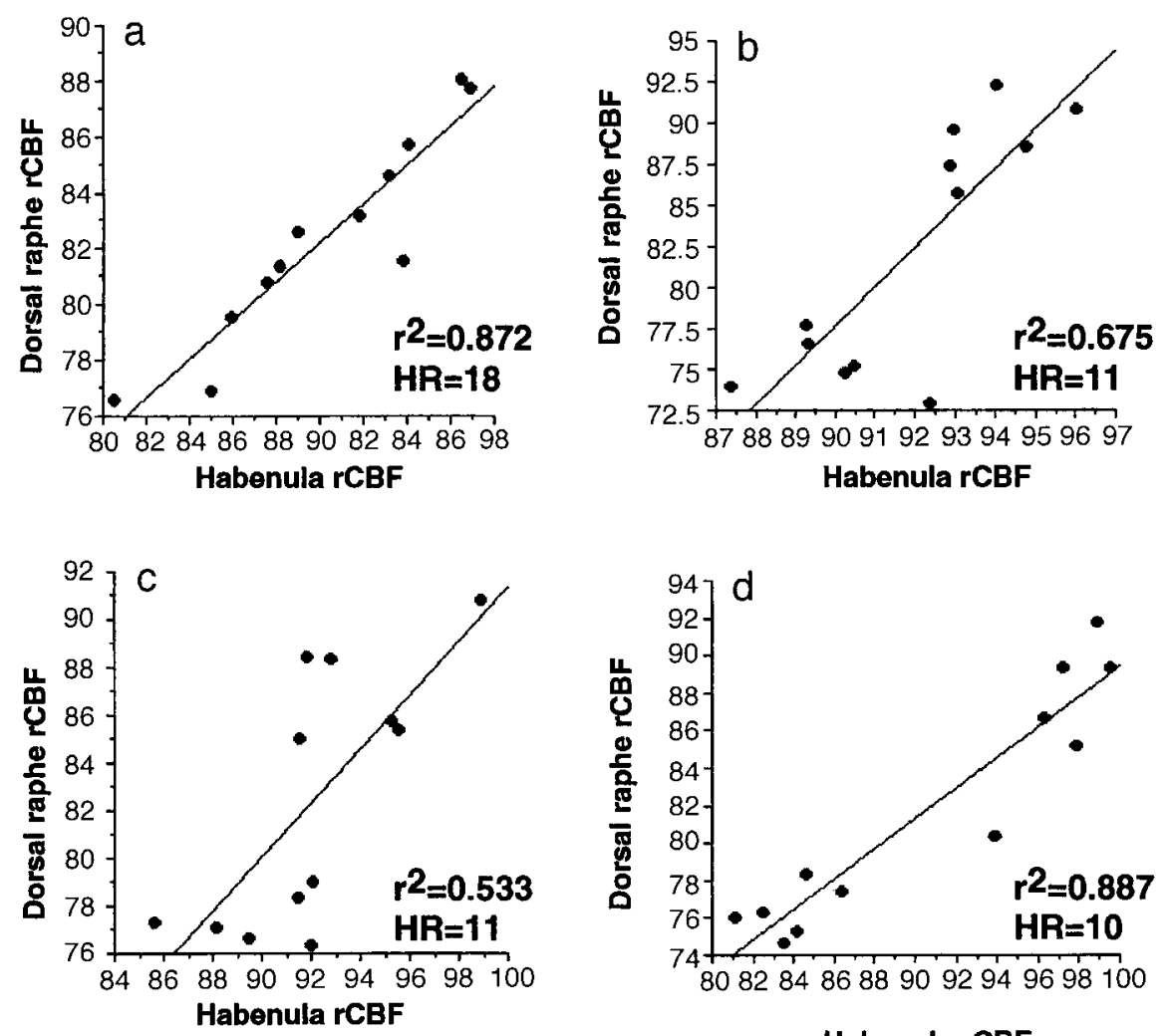

Habenula rCBF
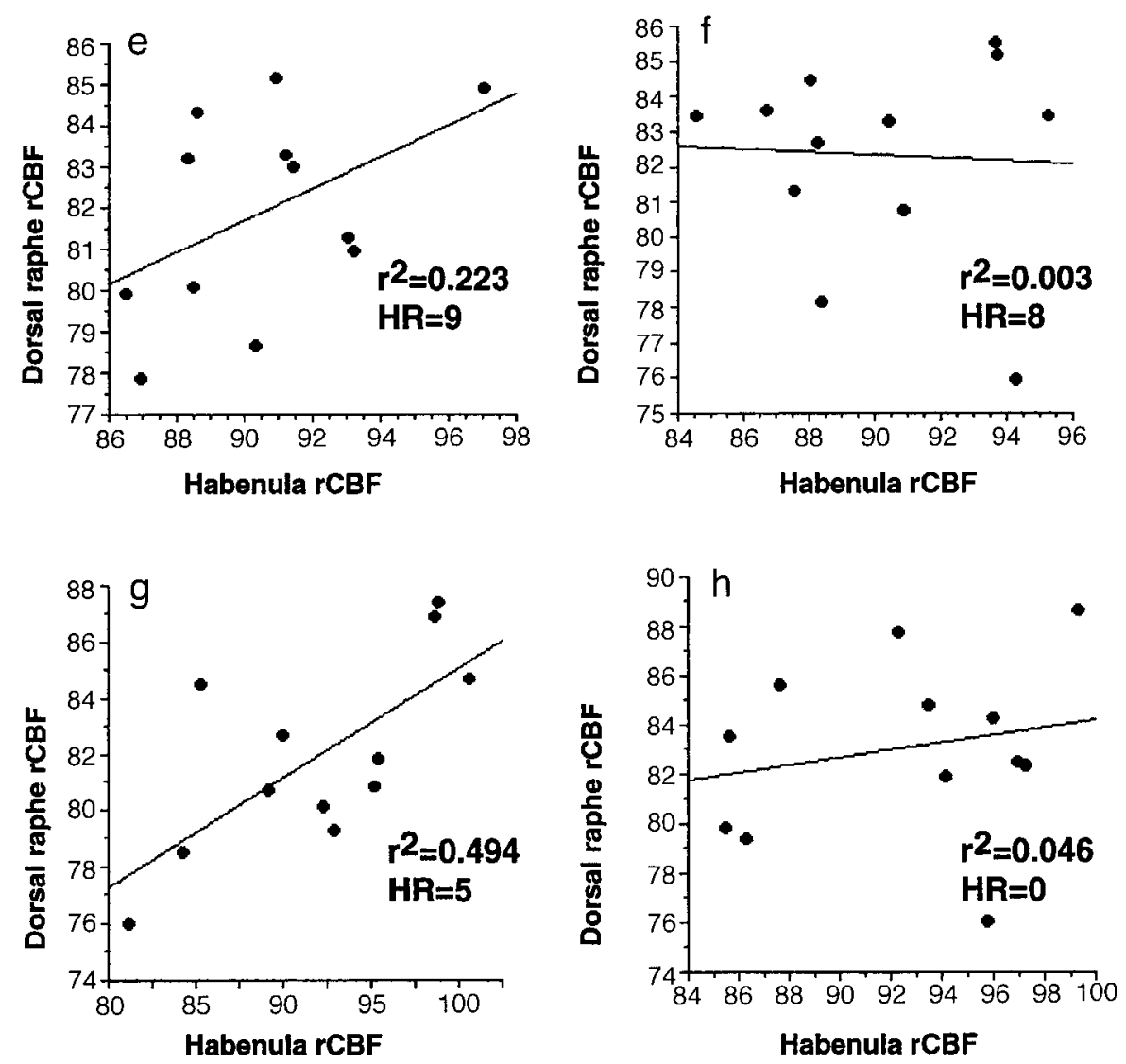

FIG. 3. Bivariateregression plots of activity in the habenula (maximal voxel $x=4, y=-24, z=6$ ) and dorsal raphénucleus (maximal voxel $x=0$, $y=-28, z=-16)$ for all eight subjects, (a-h), ranked by modified Hamilton score following tryptophan depletion. Values corresponding to each of the 12 scans are displayed. Correlation coefficients $\left(r^{2}\right)$ and modified Hamilton ratings (HR) are displayed on the regression plots for each subject. 
served, suggesting a greater balance between the two opposing types of input and a more complex, nonlinearrelationship between habenula and raphé responses (Figs. 3e-3h).

The pattern of habenula activity observed in the present study has remarkable parallels with a ${ }^{14} \mathrm{C}-2-$ deoxyglucose autoradiographic study in rats, which consistently found increased metabolism in the habenula in relation to several models of depressed behavior (Caldecott-Hazard et al., 1988). The increase in habenula activity could be reversed, however, by an antidepressant that was also effective in preventing the depressed behavior (Caldecott-Hazard et al., 1988). In addition to depression, animal studies have implicated the habenula in a wide range of complex behaviors including self-stimulation, mating, maternal behavior, sleep, ingestive behavior, naloxone-reversible analgesia, and exploration (Goldstein, 1983; Evans and Thornton, 1984; Nakajima, 1984; Mahieux and Benabid, 1987; Carvey et al., 1987). However, to our knowledge, there are no previous empirical data directly relating habenula activity to human behavior.

5-HT has been implicated in several cognitive functions that are impaired in depression (Sirvio et al., 1994), consistent with the hypothesis that serotonergic disturbance underlies affective disorders. It is significant in the present study, therefore, that task-specific neural responses were modulated by tryptophan depletion, even though performance was equivalent for balanced and depleted sessions. The modulated regions, in left amygdala and left anterior cingulate (Fig. 4), receive strong serotonergic projections from the dorsal raphé (Tork and Hornung, 1990) and have been implicated in both language generation tasks and mood disorders by previous neuroimaging studies (Bench et al., 1995; Drevets and Raichle, 1995; Baker et al., 1997). If changes in connectivity between habenula and raphé (Fig. 3) are important in controlling cer ebral 5-HT release, as seems to be indicated by animal data (Wang and Aghajanian 1977; Soubrie et al., 1977; Reisne et al., 1982), then disturbed activity in the habenula-raphé pathway may represent a common mechanism for abnormalities of mood and cognition in depression.

\section{CONCLUSION}

Our neuroimaging data reveal that the extent of mood change following tryptophan depletion is related to the covariation of activity between two serotonergic subcortical nuclei, the habenula and dorsal raphé. Highly correlated activity between these structures was present only in subjects with high postdepletion depression ratings. These data are consistent with a model of serotonergic function in which the habenula projection to the raphé constitutes a convergent feed- back pathway that controls release of cerebral 5- $\mathrm{HT}$. According to this model, the normal balance of excitation and inhibition in the habenula-raphé pathway is disturbed in depression, resulting in abnormal release of 5-HT throughout the brain. Since the habenula, via its influence on raphé activity, can affect serotonergic activity in widespread cortical and subcortical regions, disruption of its putative integrative and feedback role may provide a common underlying mechanism for the affective, behavioral, and cognitive symptoms that constitute the syndrome of depression.

\section{ACKNOWLEDGMENTS}

K.A.S. was supported by a Wellcome Trust Training Fellowship. J .S.M., R.J.D., and K.R.F. are supported by the Wellcome Trust. P.J .C. is an MRC Clinical Scientist.

\section{REFERENCES}

Aghajanian, G. K., and Wang, R. Y. 1977. Habenular and other midbrain raphé afferents demonstrated by a modified retrograde tracing technique. Brain Res. 122:229-242.

Baker, S., Frith, C. D., and Dolan, R. J . 1997. Theinteraction between mood and cognitive function studied with PET. Psychol. Med. 27:565-578.

Bench, C. J ., Frackowiak, R. S. J ., and Dolan, R. J . 1995. Changes in regional cerebral blood flow on recovery from depression. Psychol. Med. 25:247-251.

Bremner, J . D., Innis, R. B., Salomon, R. M., Staib, L. H., Ng, C. K., Miller, H. L., Bronen, R. A., Krystal, J . H., Duncan, J., Rich, D., Price, L. H., Malison, R., Dey, H., Soufer, R., and Charney, D. S. 1997. Positron emission tomography measurement of cerebral metabolic correlates of tryptophan depletion-induced depressive relapse. Arch. Gen. Psychiat. 54:364-374.

Caldecott-Hazard, S., Mazziotta, J ., and Phelps, M. 1988. Cerebral correlates of depressed behaviour in rats, visualised using ${ }^{14} \mathrm{C}-2$ deoxyglucose autradiography. J . Neurosci. 8:1951-1961.

Carvey, P. M., Kao, L. C., and Klawans, H. L. 1987. The effect of bilateral kainic acid-induced lateral habenula lesions on dopaminemediated behaviors. Brain Res. 409:193-196.

Coppen, A. 1967. The biochemistry of affective disorders. Br. J . Psychiat. 113:1237-1264.

Cowen, P. J ., and Charig, E. M. 1987. Neuroendocrine responses to intravenous tryptophan in major depression. Arch. Gen. Psychiat. 44:958-966.

Delgado, D., Charney, D. S., Price, L. H., Aghajanian, G. K., Landis, H., and Heninger, G. R. 1990. Serotonin function and the mechanism of antidepressent action. Arch. Gen. Psychiat. 47:411-418.

Drevets, W. C., and Raichle, M. E. 1995. PET studies of emotion. In The Cognitive Neurosciences (M. S. Gazzaniga, Ed.), pp. 11531164. MIT Press, Cambridge, MA.

Drevets, W. C., Videen, T. O., Price, J . L., Preskorn, S. H., Carmichael, S. T., and Raichle, M. E. 1992. A functional anatomical study of unipolar depression. J . Neurosci. 12:3628-3641.

Duvernoy, H. 1991. The Human Brain: Surface, Three Dimensional Sectional Anatomy and MRI. Springer-Verlag, Vienna.

Evans, J. A. C., and Thornton, E. W. 1984. Impaired acquisition of $\mathrm{DRL}$ operant responding following lesion of the habenula nucleus. Physiol. Psychol. 12:220-226.

Fox, P. T., Miezin, F. M., Allman, J . M., Van-Essen, D. C., Raichel, M. E., 1987. Retinotopic organization of human visual cortex mapped with positron-emission tomography. J . Neurosci. 7:913-22. 
Friston, K. J ., Büchel, C., Fink, G., Morris, J. S., Rolls, E. T., and Dolan, R. J. 1997. Psychophysiological and modulatory interactions in neuroimaging. Neurol mage 6:218-229.

Friston, K. J ., Holmes, A. P., Worsley, K. J ., Poline, J .-P., Frith, C. D., and Frackowiak, R. S. J. 1995. Statistical parametric maps in functional imaging: A general linear approach. Hum. Brain Mapp. 2:189-210.

Goldstein, R. 1983. A GABAergic habenulo-raphé pathway mediation of the hypnogenic effects of vasotocin in cat. Neuroscience 10:941945.

Heninger, G. R., Charney, D. S., and Sternberg, D. E. 1984. Serotonergic function in depression. Arch. Gen. Psychiat. 41:398-402.

Kalen, P., Strecker, R. E., Rosengren, E., and Bjorklund, A. 1989. Regulation of striatal serotonin release by the lateral habenuladorsal raphé pathway in the rat demonstrated by in vivo microdialysis: Role of excitatory amino acids and GABA. Brain Res. 492:187-202.

Mahieux, G., and Benabid, A. L. 1987. Naloxone-reversible analgesia induced by electrical stimulation of the habenula in the rat. Brain Res. 406:118-129.

Mai, J . K., Assheuer, J ., and Paxinos, G. 1997. Atlas of the Human Brain. Academic Press, San Diego.

Marburg, O. 1994. The structure and fiber connections of the human habenula. J . Comp. Neurol . 80:211-233.

Matthews-F elton, T., Corodimas, K. P., Rosenblatt, J . S., and Morrell, J. L. 1995. Lateral habenula neurons are necessary for the hormonal onset of maternal behavior and for the display of postpartum estrus in naturally parturient female rats. Behav. Neurosci. 109:1172-1188.

Meltzer, H. Y. 1990. Role of serotonin in depression. Ann. N.Y. Acad. Sci. 600:486-500.

Nakajima, S. 1984. Serotonergic mediation of habenula selfstimulation in the rat. Pharmacol. Biochem. Behav. 20:859-862.

Nauta, W. J . H. 1958. Hippocampal projections and related neural pathways to the mid-brain in the cat. Brain 81:319-340.

Nishikawa, T., and Scatton, B. 1985. Inhibitory influence of GABA on central serotonergic transmission: Raphé nuclei as the neuroana- tomical site of the GABAergic inhibition of cerebral serotonergic neurons. Brain Res. 331:81-90.

Reisne, T., Soubrie, P., Artaud, F., and Glowinski, J. 1982. I nvolve ment of lateral habenula-dorsal raphé neurons in the differential regulation of striatal and nigral serotonergic transmission in cats. J . Neurosci. 8:1962-1971.

Sakai, K., Salvert, D., Touret, M., and J ouvet, M. 1977. Afferent connections of the nucleus raphe dorsalis in the cat as visualized by the horseradish peroxidase technique. Brain Res. 137:11-35.

Sirvio, J ., Riekkinen, P., J akala, P., and Riekkinen, P. J . 1994. Experimental studies on the role of serotonin in cognition. Prog. Neurobiol. 43:363-379.

Smith, K. A., Fairburn, C. G., and Cowan, P. J . 1997. Relapse of depression after rapid depletion of tryptophan. Lancet 349:915919.

Smith, K. A., Morris, J. S., Friston, K. J ., Cowen, P. J ., and Doalan, R. J . 1999. Dissociating brain mechanisms of pathological mood and its associated cognitive impairment. B. J . Psych. 174:525-529.

Soubrie, P., Reisne, T., Artaud, F., and Glowinski, J . 1977. Role of the lateral habenula in modulating nigral and striatal in vivo $\left[{ }^{3} \mathrm{H}\right]$ serotonin release in the cat. Brain Res. 222:192-197.

Stanley, M., and Mann, J .J . 1983. I ncreased serotonin-2 binding sites in frontal cortex of suicide victims. Lancet 1(8318):214-216.

Stern, W. C., J ohnson, A., Bronzino, J . D., and Morgan, P. J . 1981. Neuropharmacology of the afferent projections from the lateral habenula and substantia nigra to the anterior raphe in the rat. Neuropharmacology 20:979-989.

Sutherland, R. J . 1982. The dorsal diencephalic conduction system: A review of the anatomy and functions of the habenular complex. Neurosci. Biochem. Rev. 6:1-13.

Talairach, J ., and Tournoux, P. 1988. Co-planar Stereotaxic Atlas of theHuman Brain. Thieme, New York.

Thorton, E. W., Evans, J. A. C., and Harris, C. 1985. Attenuated response to nomifensine in rats during a swim test following lesion of the habenula complex. Psychopharmacol ogy 87:81-85.

Tork, I., and Hornung, J.-P. 1990. Raphé nuclei and the serotonergic system. In The Human Nervous System (G. Paxinos, Ed.), pp. 1002-1022. Academic Press, San Diego.

Wang, R. Y., and Aghajanian, G. K. 1977. Physiological evidence for habenula as major link between forebrain and midbrain raphé. Science 197:89-91. 